\title{
THE DISK-WIND-JET CONNECTION IN THE BLACK HOLE H 1743-322
}

\author{
J. M. Miller ${ }^{1}$, J. Raymond ${ }^{2}$, A. C. Fabian ${ }^{3}$, C. S. Reynolds ${ }^{4}$, A. L. King ${ }^{1}$, T. R. Kallman ${ }^{5}$ E. M. Cackett $^{6}$, \\ M. VAN DER KLIS ${ }^{7}$, AND D. T. H. STEEGHS ${ }^{8}$ \\ ${ }^{1}$ Department of Astronomy, University of Michigan, 500 Church Street, Ann Arbor, MI 48109-1042, USA; jonmm@umich.edu \\ ${ }^{2}$ Smithsonian Astrophysical Observatory, 60 Garden Street, Cambridge, MA 02138, USA \\ ${ }^{3}$ Institute of Astronomy, University of Cambridge, Madingley Road, Cambridge CB3 OHA, UK \\ ${ }^{4}$ Department of Astronomy, University of Maryland, College Park, MD 20742, USA \\ ${ }^{5}$ Laboratory for High Energy Astrophysics, NASA Goddard Space Flight Center, Code 662, Greenbelt, MD 20771, USA \\ ${ }^{6}$ Department of Physics and Astronomy, Wayne State University, 666 West Hancock Street, Detroit, MI 48201, USA \\ ${ }^{7}$ Astronomical Institute "Anton Pannekoek," University of Amsterdam, Science Park 904, 1098-XH, Amsterdam, The Netherlands \\ ${ }^{8}$ Department of Physics, University of Warwick, Coventry CV4 7AL, UK \\ Received 2012 May 7; accepted 2012 August 21; published 2012 October 10
}

\begin{abstract}
X-ray disk winds are detected in spectrally soft, disk-dominated phases of stellar-mass black hole outbursts. In contrast, compact, steady, relativistic jets are detected in spectrally hard states that are dominated by non-thermal $\mathrm{X}$-ray emission. Although these distinctive outflows appear to be almost mutually exclusive, it is possible that a disk wind persists in hard states but cannot be detected via X-ray absorption lines owing to very high ionization. Here, we present an analysis of a deep, 60 ks Chandra/HETGS observation of the black hole candidate H 1743-322 in the low/hard state. The spectrum shows no evidence of a disk wind, with tight limits, and within the range of ionizing flux levels that were measured in prior Chandra observations wherein a wind was clearly detected. In H 1743-322, at least, disk winds are actually diminished in the low/hard state, and disk winds and jets are likely state dependent and anti-correlated. These results suggest that although the launching radii of winds and jets may differ by orders of magnitude, they may both be tied to a fundamental property of the inner accretion flow, such as the mass accretion rate and/or the magnetic field topology of the disk. We discuss these results in the context of disk winds and jets in other stellar-mass black holes, and possible launching mechanisms for black hole outflows.
\end{abstract}

Key words: accretion, accretion disks - black hole physics - X-rays: binaries

Online-only material: color figures

\section{INTRODUCTION}

Recent Chandra/HETGS observations of stellar-mass black holes and neutron stars have revealed disk winds through blueshifted X-ray absorption lines (Miller et al. 2006a, 2006b, 2008; Kubota et al. 2007; Ueda et al. 2009; Neilsen \& Lee 2009; King et al. 2012a, 2012b; Ponti et al. 2012). Such winds are not a negligible part of the accretion flow: estimates for the mass outflow rate in winds range from a fraction of the accretion rate through the disk to many times greater than the mass accretion rate $(\dot{m})$ through the disk. A full understanding of disk accretion now requires an understanding of such outflows and how they are driven. More broadly, these winds may provide an important grounding for tentative evidence of ionized winds from the inner disk of active galactic nucleus (Tombesi et al. 2010; King et al. 2012a, 2012b).

Stellar-mass black hole disk winds appear to be state dependent: they are detected in spectrally soft, disk-dominated states, but are not clearly detected in the "low/hard" state (Miller et al. 2006b, 2008; Neilsen \& Lee 2009; Blum et al. 2010; Ponti et al. 2012; King et al. 2012a). In contrast, relativistic radio jets are ubiquitous in the "low/hard" state (Fender et al. 2004), but quenched in disk-dominated soft states (e.g., Russell et al. 2011). Thus, it is possible that winds and jets are anti-correlated, but related.

An anti-correlation might offer rare clues to the mechanisms that drive wind and jets. However, the nature of changes to the accretion inflow geometry and radiative processes across state transitions remains unclear (e.g., Esin et al. 1997; Reis et al. 2010). Even the apparent absence of disk winds in the "low/hard" state may only be an observational effect driven by overionization of the gas. A wind might continue unabated, but simply be impossible to detect through absorption lines owing to a higher ionizing photon flux level.

Ponti et al. (2012) suggested that a higher ionizing flux could not explain the lack of observable wind features in the hard state. However, they did not consider whether the associated change in spectral shape could provide the required change in ionization. Neilsen \& Homan (2012) carried out a more complete analysis to show that the extremely dense wind seen in the soft state of GRO J1655-40 by Miller et al. (2006a) could not also be present in a harder state (likely an "intermediate" state) observed a few days earlier, with the difference in absorption lines explained by ionization alone. That extreme wind state has only been reported in one other black hole binary observation, and the driving mechanism may be different from that of the more normal winds in which only Fe XXVI and perhaps Fe XXV are detectable.

In this Letter, we compare the Fe absorption lines seen in the soft state of H1743-322 with the lack of absorption lines in a true low/hard state. We find that the difference in photoionization rate cannot account for the spectra, but that the wind must be genuinely suppressed. We use a quantitative approach to the difference in absorption line strengths to reach a better understanding of disk winds based on analysis of a deep Chandra HETGS observation of H 1743-322 in the low/hard state, focusing on the physical parameters implied by the apparent absence of the wind.

\section{OBSERVATIONS AND DATA REDUCTION}

During its 2003 outburst, H 1743-322 was observed simultaneously using the Chandra/HETGS and RXTE on four 
occasions (Miller et al. 2006b). All four of those observations captured relatively soft flux states. Three could be roughly classified as "high/soft" states. The remaining observation (second in the sequence) likely captured a harder (but still luminous) "very high" state.

H 1743-322 was again observed with the Chandra/HETGS and RXTE in 2010. The Chandra observation started on 2010 August 8 at 23:03:48 UT and was $60.5 \mathrm{ks}$ in duration. RXTE observed H 1743-322 simultaneously with Chandra, starting on 2010 August 9 at $05: 35: 51$ UT for a total duration of $6.1 \mathrm{ks}$. Rapid analysis of the RXTE observation confirmed that H 1743-322 was in a "low/hard" state at the time of these observations (Belloni et al. 2010).

The Chandra High Energy Transmission Gratings (HETG) were used to disperse the incident flux onto the Advanced CCD Imaging Spectrometer "spectroscopic array" (ACIS-S). To prevent photon pile-up, the ACIS-S array was operated in continuous clocking or "GRADED_CC" mode, which reduced the frame time to $2.85 \mathrm{~ms}$. For a discussion of this mode, please see Miller et al. (2006b). All Chandra data reduction was accomplished using CIAO version 4.4. Time-averaged firstorder HEG and MEG spectra were extracted from the Level-2 event file. Redistribution matrix files ( $\mathrm{rmfs}$ ) were generated using the tool "mkgrmf"; ancillary response files (arfs) were generated using "mkgarf." The first-order HEG spectra and responses were combined using "add_grating_orders." The spectra were grouped to require a minimum of 10 counts bin ${ }^{-1}$.

The standard RXTE pipeline spectral files and responses for the Proportional Counter Array (PCA) and HEXTE were downloaded from the HEASARC archive, and employed for spectral fitting. A systematic error of $0.6 \%$ was added to the PCA spectrum in quadrature. All spectral analyses were conducted using XSPEC version 12.6.0. All errors quoted in this Letter are $1 \sigma$ errors.

\section{ANALYSIS AND RESULTS}

The central questions in this Letter require estimates of the ionizing flux and column density in each observation

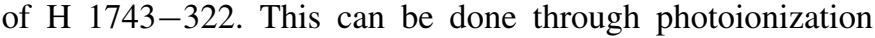
modeling, but a simpler and more direct approach is to measure the equivalent width of absorption lines since $\mathrm{EW} \propto N$ when the absorbing gas is on the linear part of the curve of growth.

\subsection{The Spectral Continuum in the 2010 Low/Hard State}

We fit the combined first-order Chandra/HEG and RXTE/PCA spectra of H 1743-322 jointly. The HEG spectrum was fit limited to the $1.2-9.0 \mathrm{keV}$ band, owing to calibration uncertainties and poor signal on either side of this range. The PCA spectrum was fit in the $3.0-30.0 \mathrm{keV}$ band, again owing to calibration uncertainties on either side. In the joint fits, a simple constant was allowed to float between the spectra to account for differences in the flux calibrations.

A fit with a simple absorbed power-law model with $\Gamma=$ $1.77 \pm 0.01$ does not give a formally acceptable fit $\left(\chi^{2} / v=\right.$ 1.56), but it does characterize the flux well. It is likely that the poor fit is driven by uncertainties in the cross-calibration of the instruments. When each spectrum is permitted to derive its own power-law index in a joint fit, a value of $\Gamma=1.93 \pm 0.01$ is found for the HEG spectrum while $\Gamma=1.71 \pm 0.01$ is found for the $R X T E / \mathrm{PCA}$ spectrum, and a much better fit is derived $\left(\chi^{2} / v=1.13\right)$. If the steeper index is assumed to be the right time-averaged value for the lengthy Chandra observation, it

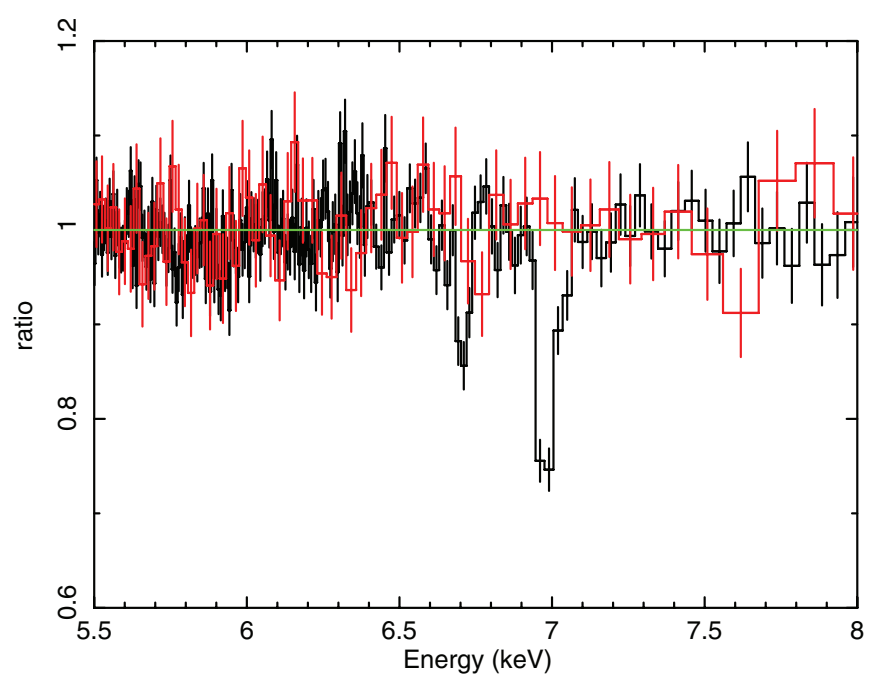

Figure 1. Line spectra from two Chandra/HETGS observations of H 1743-322. The data have been divided by a simple continuum and binned for visual clarity. The observation in black was obtained in a disk-dominated phase; it is listed as "Observation 1" in Miller et al. (2006b). A disk wind was detected through the blueshifted Fe XXV and Fe XXVI absorption lines in that spectrum. The deep low/hard state observation considered in this paper is shown in red. No significant absorption lines are evident, and restrictive upper limits are obtained through direct fits.

(A color version of this figure is available in the online journal.)

leads to a low value for the $8.8-30 \mathrm{keV}$ ionizing flux (see below). To be conservative, then, we simply adopt the power-law index derived in the joint fit $(\Gamma=1.77)$ as an approximate value, and derive the unabsorbed $8.8-30 \mathrm{keV}$ flux based on that model.

\subsection{Limits on Absorption Lines in the 2010 Hard State}

To test for the presence of Fe XXV and Fe XXVI absorption lines in the low/hard state spectrum of $\mathrm{H} \mathrm{1743-322,} \mathrm{we} \mathrm{added}$ Gaussians at $1.850 \AA$ and $1.780 \AA(6.700 \mathrm{keV}$ and $6.970 \mathrm{keV}$, respectively; Verner et al. 1996). The range of line widths and velocity shifts measured in the line detections reported in Miller et al. (2006b) was sampled in order to ensure consistency and conservative limits. We measure $90 \%$ confidence upper limits of $\mathrm{EW} \leqslant 0.58 \mathrm{~m} \AA$ and $\mathrm{EW} \leqslant 0.42 \mathrm{~m} \AA$ for Fe XXV and Fe XXVI, respectively. These limits are a factor of several lower than the line detections reported in Miller et al. (2006b). Figure 1 shows data/model ratio spectra from a prior Chandra observation and the low/hard state considered here.

Larger potential velocity shifts were also examined, since one means of increasing the ionization of a gas is to accelerate it. A potential feature is apparent at $1.834 \AA(6.760 \mathrm{keV})$. However, this feature is merely noise: first, the feature is not significant at even the $2 \sigma$ level; second, it is unlikely that Fe XXV would be observed in the absence of Fe XXVI (see, e.g., Kallman \& McCray 1982), especially if the gas is potentially more highly ionized than when both were detected. There is no evidence for an Fe XXVI line at a velocity shift required if the feature at the $6.760 \mathrm{keV}$ line is identified with Fe Xxv.

Limits on the line equivalent widths obtained with Chandra (again, $0.58 \mathrm{m \AA}$ and $0.42 \mathrm{m \AA}$ for Fe XXV and Fe XXVI, respectively) are tighter than those obtained in a prior Suzaku observation in the low/hard state $(0.97 \mathrm{m \AA}$ and $0.64 \mathrm{~m} \AA$; Blum et al. 2010). Importantly, whereas the ionizing flux derived from the Suzaku observation was higher than during the prior observations wherein a disk wind was detected, the ionizing flux derived 


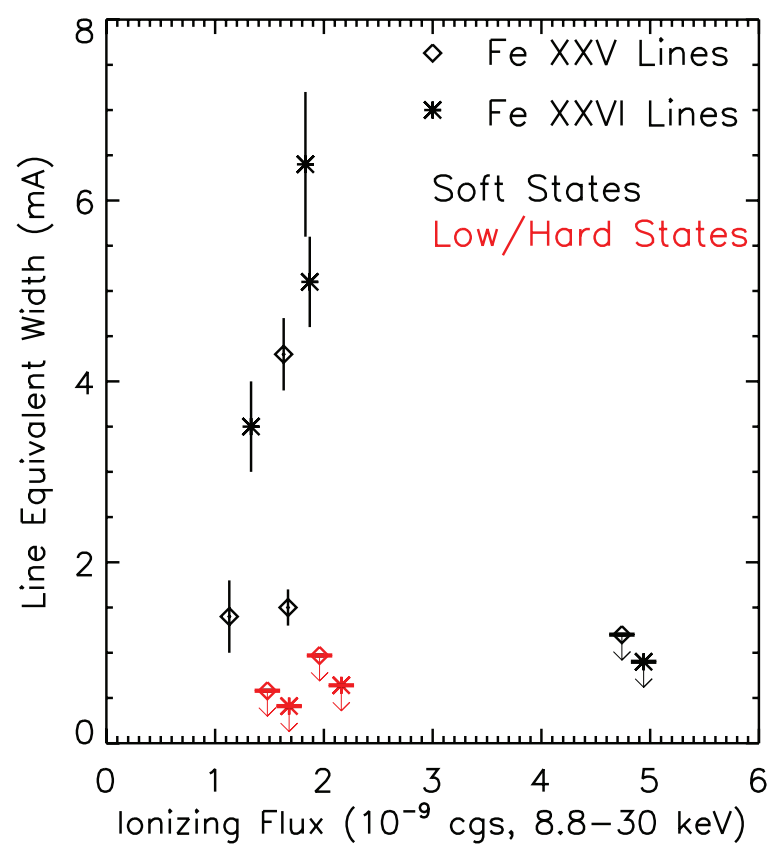

Figure 2. Blueshifted Fe XXV and Fe XXVI lines are the primary tracers of disk winds in stellar-mass black hole spectra. The figure above plots line equivalent width (a proxy for the column density) vs. the ionizing flux, based on Chandra observations of H 1743-322. Points corresponding to Fe XXVI were artificially shifted upward in ionizing flux by 0.2 for visual clarity. Upper limits $(90 \%$ confidence) are characterized with arrows. Black points represent measurements obtained in soft, disk-dominated states while red points signify measurements made in the low/hard state. Line limits from a Suzaku low/hard state observation (Blum et al. 2010) are also included (the higher flux pairing of points).

(A color version of this figure is available in the online journal.)

in the new Chandra observation is lower than two of the three prior observations with detections.

\subsection{Line Equivalent Widths versus Ionizing Flux}

In order to ionize He-like Fe Xxv, photons with $E \geqslant 8.8 \mathrm{keV}$ are required $(E \geqslant 9.3 \mathrm{keV}$ is required for Fe XxVI; Verner et al. 1996). To characterize the ionizing flux in each observation

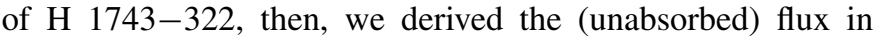
the $8.8-30.0 \mathrm{keV}$ band, based on direct fits to the spectral continuum. For plausible spectral indices, the $30-100 \mathrm{keV}$ photon flux represents $\leqslant 1 \%$ of the photoionization rate. Given that some low/hard state spectra have a spectral break at approximately $30 \mathrm{keV}$, and given that photons above $30 \mathrm{keV}$ are unimportant, the $8.8-30.0 \mathrm{keV}$ band is a meaningful and pragmatic range for characterizing the ionizing flux.

Spectral fits to Chandra and RXTE observations in bright states are described in Miller et al. (2006b). These models were used to calculate the unabsorbed $8.8-30 \mathrm{keV}$ flux for each bright phase observation. Where multiple RXTE observations were made within a single Chandra exposure, their ionizing flux values were averaged. The same procedure was used to calculate the ionizing flux in the Suzaku low/hard state observation (Blum et al. 2010). For the Chandra low/hard state observation that is the focus of this Letter, the unabsorbed 8.8-30 keV flux was calculated based on the fits in Section 3.1.

Figure 2 plots line equivalent widths versus the $8.8-30.0 \mathrm{keV}$ ionizing flux, based on Chandra detections and limits. The Chandra upper limits in the "low/hard" state reported in this work are lower than the ionizing flux levels in two of three prior cases wherein a disk wind was detected (Miller et al. 2006b). It is probable, then, that the wind is diminished or even quenched in the "low/hard" state.

The strongest Fe XXVI line detected in the 2003 outburst corresponded to $N_{\mathrm{XXVI}}=5.5 \pm 0.7 \times 10^{17} \mathrm{~cm}^{-2}$ or $N_{\mathrm{H}}=1.8 \times$ $10^{23} \mathrm{~cm}^{-2}$, after accounting for the abundance of Fe $\left(3 \times 10^{-5}\right.$ is typical of the literature) and ionization fraction (Fe XXVI/ Fe XXVII $\leqslant 0.1$; e.g., Kallman \& McCray 1982). In simple terms, then, the new observation implies $N_{\mathrm{H}} \leqslant 0.86 \times 10^{22} \mathrm{~cm}^{-2}$. In the high ionization limit, e.g., when FeXXV is not seen, $f_{\mathrm{XXVI}} \propto n q_{\mathrm{rec}} / L_{X} r^{2}$ (where $f_{\mathrm{XXVI}}$ is the ionization fraction of Fe XXVI, $n$ is the number density, and $q_{\text {rec }}$ is the recombination rate coefficient). Thus, if $r$ stays the same, $f \propto n$. Therefore, reducing the density by a factor of $\sim 3$ would reduce the total column by a factor of $\sim 3$, and the corresponding reduction in $\xi$ would reduce the ionization fraction $(\mathrm{Fe} X X V I / F e)$ by $\sim 3$, in combination giving an order of magnitude reduction in $N_{\mathrm{XXVI}}$ (the observed quantity). This would require $\dot{M}_{\text {wind }}$ to be at least a factor of $\sim 3$ lower. Note that the strongest line detected in 2003 did not show a significant blueshift; if the outflow velocity is tied to the ionizing luminosity, the low ionizing flux measured in the deep low/hard state observation could imply an even lower outflow rate since $\dot{M}_{\text {wind }} \propto v$, and a lower kinetic power since $L_{\text {wind }} \propto v^{3}$.

If a strong wind did persist in the low/hard state, it would have to originate very close to the black hole. The photoionization models presented in Miller et al. (2006b) suggest $\xi=3-4 \times$ $10^{5} \mathrm{erg} \mathrm{cm} \mathrm{s}^{-1}$, but this could potentially be pushed lower, and a reasonable lower bound would be $\xi \geqslant 10^{4}$. For a distance of $8.5 \mathrm{kpc}$ (Steiner et al. 2012), the flux measured in the low/hard state corresponds to $L \simeq 2 \times 10^{37} \mathrm{erg} \mathrm{s}^{-1}$. If the low $/$ hard state wind is at least as ionized as before, and taking $\xi=L / n r^{2}$, $\left(2 \times 10^{37} \mathrm{erg} \mathrm{s}^{-1}\right) / n r^{2} \geqslant 10^{4} \mathrm{erg} \mathrm{cm} \mathrm{s}^{-1}$. Assuming $N=n r$, then $N \leqslant\left(2 \times 10^{33} \mathrm{~cm}^{-1}\right) / r$. If the wind were undiminished, $N=10^{23} \mathrm{~cm}^{-2}$. This would imply $r=10^{10} \mathrm{~cm}$ and $n=$ $10^{13} \mathrm{~cm}^{-3}$. Such a small radius and high density are similar to the magnetic wind detected in GRO J1655-40 (Miller et al. 2006a, 2008; Kallman et al. 2009, also see Neilsen \& Homan 2012). Moreover, Luketic et al. (2010) have recently shown that winds with $n \geqslant 10^{12} \mathrm{~cm}^{-3}$ are difficult or impossible to drive thermally.

\section{DISCUSSION AND CONCLUSIONS}

A dense, equatorial disk wind was previously detected in Chandra/HETGS observations of $\mathrm{H} \mathrm{1743-322}$ in diskdominated states (Miller et al. 2006b). This source is a strong black hole candidate based on its X-ray properties (e.g., Homan et al. 2005), but its mass has not yet been determined. Assuming fiducial values of $d=8.5 \mathrm{kpc}$ (Steiner et al. 2012; based on the proximity of the source to the Galactic center) and $M=10 M_{\odot}$, the photoionization models applied in Miller et al. (2006b) describe a wind with $\dot{M}_{\text {out }}=(3-4) \times 10^{17} \mathrm{~g} \mathrm{~s}^{-1}$ that may originate within $r \simeq 10^{2}-10^{3} G M / c^{2}$ of the black hole.

The blueshifted Fe XXV and Fe XXVI absorption lines found in the brighter, disk-dominated phases are not detected in this deep observation. Importantly, the ionizing photon flux in this "low/hard" state was found to be within the range measured when a disk wind was previously detected. Using simple arguments, we have shown that the mass outflow rate is likely reduced by at least a factor of $\sim 3$ in the low/hard state, compared to the strongest prior line detections, and might be reduced far more severely. If a wind with a column density like that measured previously were to persist in the low/hard state, it would have to be dense and originate close to the black hole, 
and would likely be driven magnetically (e.g., Miller et al. 2008; Luketic et al. 2010).

A disk wind was absent in $\mathrm{H} \mathrm{1743-322} \mathrm{during} \mathrm{one} \mathrm{of}$ the Chandra observations in 2003 (see the rightmost limits in Figure 2). Based on photoionization modeling, Miller et al. (2006b) concluded that the non-detection likely required a geometric change (lower density, depth, or covering factor), not merely higher ionization. Similarly, Neilsen \& Homan (2012) recently concluded that overionization could not account for variability seen in the disk wind in GRO J1655-40. Stellarmass black hole winds may be thermally driven: radiation from the central engine heats gas in the outer disk to the local escape speed (see, e.g., Begelman et al. 1983; Woods et al. 1996; also see Luketic et al. 2010). It is not clear that such winds should be strongly variable. Geometric changes associated with state transitions - such as the presence or absence of an inner disk (e.g., Esin et al. 1997; also see Reis et al. 2010)—need not affect the outer disk. The important parameter for wind detection is the ionizing photon flux, which need not change drastically across states.

Ueda et al. (2010) suggested a geometric change that might explain variability in thermal winds: the development of a hot, geometrically thick, optically thick $(\tau=7-10)$ corona that can "shield" the outer disk. This picture may be consistent with the "very high" state and may be able to account for the prior nondetection of a disk wind in H 1743-322 (Miller et al. 2006b). However, this geometrical change is not consistent with the "low/hard" state, which is of much greater interest since this is the only state where jets are produced in a steady fashion (e.g., Fender et al. 2004). Black hole spectra in the "low/hard" state require a relatively low optical depth and high temperature when fit with Componization models $\left(\tau \leqslant 1-2, k T_{e}=30-120 \mathrm{keV}\right.$; see, e.g., Gierlinski et al. 1997; Torii et al. 2011). Moreover, the outer disk must be irradiated in order to explain UV and optical emission in the "low/hard" state (e.g., Rykoff et al. 2007; Reynolds \& Miller 2012).

Magnetic driving may provide an alternative to thermal driving and may be suited to the anti-correlation between winds and jets when comparing "high/soft" and "low/hard" states. For instance, the magnetic field configuration may change from toroidal to poloidal in transitions from disk-dominated states to the "low/hard" state. Disk winds might then be driven by magnetic pressure generated in a thin disk (e.g., Proga 2003; Ohsuga et al. 2009) while jets might be driven by magnetocentrifugal acceleration along poloidal field lines (Blandford \& Payne 1982), perhaps aided by black hole spin (Blandford \& Znajek 1977). This change could be precipitated by a drop in $\dot{m}$ through the disk; poloidal fields may be easier to anchor in thicker disks (Reynolds et al. 2006). Alternatively, it is possible that poloidal fields could dominate on each side of a state transition, and that $\dot{m}_{\text {disk }}$ modulates how much mass is loaded onto poloidal field lines (e.g., Spruit 1996). Winds would originate when the mass outflow rate is high, perhaps breaking field lines or dragging them to make a small angle with respect to the disk (Proga 2003). Jets would then originate when the mass outflow rate is relatively low, consistent with the "low/hard" state, allowing for more effective acceleration. This latter scenario may be supported by recent work suggesting that black hole winds and jets may be regulated in a common fashion across the mass scale (King et al. 2012b).

The analysis of wind properties across state transitions presented in this work may favor a magnetic wind component. Stronger support for magnetically driven winds may derive from photoionization modeling that is able to infer a small launching radius and/or very high mass outflow rate (e.g., Miller et al. 2008), or perhaps from evidence of common regulation of the kinetic power in winds and jets (e.g., King et al. 2012b). To constrain the launching radius, the density of the gas must be measured directly, since $r^{2}=L / n \xi$. Currently, this has only been possible for GRO J1655-40, via the detection of the density-sensitive Fe XXII line pair $\left(n=10^{14} \mathrm{~cm}^{-3}\right)$, and possibly for NGC 4051 (King et al. 2012a). In contrast, the density of the disk winds in H 1743-322 and GRS 1915+105 has not been directly constrained (but estimates based on variability are given in Neilsen et al. 2011, 2012). Trends in these sources may or may not be consistent with thermal driving (Ponti et al. 2012; King et al. 2012b). Observing stellar-mass black holes with low line-of-sight column densities will facilitate radius and density constraints through the detection of Fe XXII lines, and help to better reveal wind launching mechanisms.

We thank the anonymous referee. We thank Harvey Tananbaum for executing this observation. A.L.K. acknowledges support through the NASA Earth and Space Sciences Fellowship. J.M.M. acknowledges support through the Chandra Guest Observer program.

\section{REFERENCES}

Begelman, M. C., McKee, C. F., \& Shields, 1983, ApJ, 271, 70 Belloni, T., Munoz-Darias, T., Motta, S., Stiele, H., \& Carbone, D. 2010, ATel, 2788

Blandford, R. D., \& Payne, D. G. 1982, MNRAS, 199, 883 Blandford, R. D., \& Znajek, R. L. 1977, MNRAS, 179, 433

Blum, J. L., Miller, J. M., Cackett, E., et al. 2010, ApJ, 713, 1244 Esin, A. A., McClintock, J. E., \& Narayan, R. 1997, ApJ, 489, 865 Fender, R. P., Belloni, T., \& Gallo, E. 2004, MNRAS, 355, 1105 Gierlinski, M., Zdziarski, A. A., Done, C., et al. 1997, MNRAS, 288, 958

Homan, J., Miller, J. M., Wijnands, R., et al. 2005, ApJ, 623, 383

Kallman, T. R., Bautista, M. A., Goriely, S., et al. 2009, ApJ, 701, 865 Kallman, T. R., \& McCray, R. 1982, ApJS, 50, 263

King, A. L., Miller, J. M., \& Raymond, J. C. 2012a, ApJ, 746, 2

King, A. L., Miller, J. M., Raymond, J., et al. 2012b, ApJ, submitted (arXiv:1205.4222)

Kubota, A., Dotani, T., Cottam, J., et al. 2007, PASJ, 59S, 185

Luketic, S., Proga, D., Kallman, T. R., Raymond, J. C., \& Miller, J. M. 2010, ApJ, 719,515

Miller, J. M., Raymond, J., Fabian, A., et al. 2006a, Nature, 441, 953

Miller, J. M., Raymond, J., Homan, J., et al. 2006b, ApJ, 646, 394

Miller, J. M., Raymond, J., Reynolds, C. S., et al. 2008, ApJ, 680, 1359

Neilsen, J., \& Homan, J. 2012, ApJ, 750, 27

Neilsen, J., \& Lee, J. C. 2009, Nature, 458, 481

Neilsen, J., Remillard, R., \& Lee, J. C. 2011, ApJ, 737, 69

Neilsen, J., Remillard, R., \& Lee, J. C. 2012, ApJ, 750, 71

Ohsuga, K., Mineshige, S., Mori, M., \& Kato, Y. 2009, PASJ, 61, 7

Ponti, G., Fender, R. P., Begelman, M. C., et al. 2012, MNRAS, 422, L11

Proga, D. 2003, ApJ, 585, 406

Reis, R. C., Fabian, A. C., \& Miller, J. M. 2010, MNRAS, 402, 836

Reynolds, C. S., Garofalo, D., \& Begelman, M. C. 2006, ApJ, 651, 1023

Reynolds, M. T., \& Miller, J. M. 2012, ApJ, submitted

Russell, D. M., Miller-Jones, J., Maccarone, T., et al. 2011, ApJ, 739, L19

Rykoff, E. S., Miller, J. M., Steeghs, D., \& Torres, M. A. P. 2007, ApJ, 666, 1129

Spruit, H. 1996, in Evolutionary Processes in Binary Stars, ed. R. A. M. J. Wijers, M. B. Davies, \& C. A. Tout (NATO ASI Series; Dordrecht: Kluwer) (arXiv:astro-ph/9602022)

Steiner, J. F., McClintock, J. E., \& Reid, M. J. 2012, ApJ, 745, L7

Tombesi, F., Cappi, M., Reeves, J. N., et al. 2010, A\&A, 521, 57

Torii, S., Yamada, S., Makishima, K., et al. 2011, PASJ, 63, S771

Ueda, Y., Honda, K., Takahashi, H., et al. 2010, ApJ, 713, 257

Ueda, Y., Yamaoka, K., \& Remillard, R. 2009, ApJ, 695, 888

Verner, D. A., Verner, E. M., \& Ferland, G. J. 1996, At. Data Nucl. Data Tables, 64,1

Woods, D. T., Klein, R. I., Castor, J. I., McKee, C. F., \& Bell, J. B. 1996, ApJ, 461,767 